\title{
Toxicity and transformation of graphene oxide and reduced graphene oxide in bacteria biofilm
}

\author{
Zhiling Guo a,b $^{\text {, }}$ Changjian Xie ${ }^{\mathrm{b}}$, Peng Zhang ${ }^{\mathrm{b}, *}$, Junzhe Zhang ${ }^{\mathrm{a}}$, Guohua Wang ${ }^{\mathrm{b}}$, Xiao He ${ }^{\mathrm{b}}$, Yuhui Ma ${ }^{\mathrm{b}}$, \\ Bin Zhao ${ }^{\mathrm{a}, *}$, Zhiyong Zhang ${ }^{\mathrm{b}, *}$ \\ a State Key Laboratory of Environmental Chemistry and Ecotoxicology, Research Center for Eco-Environmental Sciences, Chinese Academy of Sciences, Beijing 100085, China \\ ${ }^{\mathrm{b}}$ Key Laboratory for Biological Effects of Nanomaterials and Nanosafety, Institute of High Energy Physics, Chinese Academy of Sciences, Beijing 100049, China
}

\section{H I G H L I G H T S}

- GO and rGO showed different impacts on bacterial biofilm.

- Oxidative stress were involved in the toxicity of rGO to biofilm.

- Toxicity of rGO to biofilm were eliminated in mature phase of bacterial biofilm.

- EPS protection and oxidation of rGO contributed to the elimination of the toxicity of rGO.

\section{G R A P H I C A L A B S T R A C T}

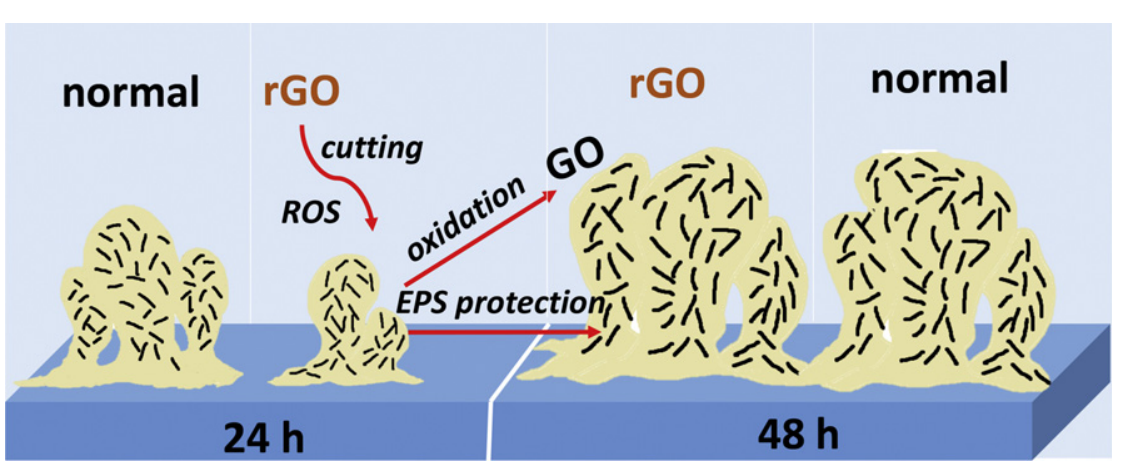

\section{A R T I C L E I N F O}

\section{Article history:}

Received 7 November 2016

Received in revised form 12 December 2016

Accepted 13 December 2016

Available online 18 December 2016

Editor: J Jay Gan

\section{Keywords:}

Graphene oxide

Reduced graphene oxide

Biofilm

Toxicity

Transformation

\begin{abstract}
A B S T R A C T
Impact of graphene based material (GNMs) on bacteria biofilm has not been well understood yet. In this study, we compared the impact of graphene oxide (GO) and reduced graphene oxide ( $\mathrm{rGO}$ ) on biofilm formation and development in Luria-Bertani (LB) medium using Escherichia coli and Staphylococcus aureus as models. GO significantly enhanced the cell growth, biofilm formation, and biofilm development even up to a concentration of $500 \mathrm{mg} / \mathrm{L}$. In contrast, rGO ( $\geq 50 \mathrm{mg} / \mathrm{L})$ strongly inhibited cell growth and biofilm formation. However, the inhibitory effects of $\mathrm{rGO}(50 \mathrm{mg} / \mathrm{L}$ and $100 \mathrm{mg} / \mathrm{L})$ were attenuated in the mature phase $(>24 \mathrm{~h})$ and eliminated at $48 \mathrm{~h}$. $\mathrm{GO}$ at $250 \mathrm{mg} / \mathrm{L}$ decreased the reactive oxygen species (ROS) levels in biofilm and extracellular region at mature phase. ROS levels were significantly increased by rGO at early phase, while they returned to the same levels as control at mature phase. These results suggest that oxidative stress contributed to the inhibitory effect of rGO on bacterial biofilm. We further found that supplement of extracellular polymeric substances (EPS) in the growth medium attenuated the inhibitory effect of rGO on the growth of developed biofilm. XPS results showed that rGO were oxidized to GO which can enhance the bacterial growth. We deduced that the elimination of the toxicity of rGO at mature phase was contributed by EPS protection and the oxidation of rGO. This study provides new insights into the interaction of GNMs with bacteria biofilm.
\end{abstract}

C 2016 Elsevier B.V. All rights reserved.

\footnotetext{
* Corresponding authors.

E-mail addresses: pengzhang@ihep.ac.cn (P. Zhang), binzhao@rcees.ac.cn (B. Zhao), zhangzhy@ihep.ac.cn (Z. Zhang).
}

\section{Introduction}

GNMs are among the most promising engineered nanomaterials (ENMs) due to their unique electronic, thermal and mechanical 
properties (Kemp et al., 2013; Goenka et al., 2014; Avouris, 2010). Application of graphene incorporated products will bring significant economic and environmental benefits; however, mass production of these compounds will inevitably increase the likelihood of their release into the environment. Given their unique physicochemical properties, GNMs may interact with different environmental organisms and pose potential hazards to environment (Hu and Zhou, 2013). Microorganism, which are ubiquitous in environment, are specifically vulnerable to the impact of GNMs, since the antibacterial property of GNMs has been evidenced by a number of studies (Zou et al., 2016). Introducing of GNMs in environment may influence the microbial community structure and activity, and potentially affect the balance of the whole ecosystem (Ahmed and Rodrigues, 2013; Combarros et al., 2016).

GO and rGO have been reported to exhibit antibacterial property to bacteria. For example, GO and rGO respectively induced 90\% and 75\% loss of viability of Escherichia coli (E. coli) and Staphylococcus aureus (S. aureus) after $4 \mathrm{~h}$ incubation (Liu et al., 2011). Similar results were also observed when exposed to GO nanowalls (Akhavan and Ghaderi, 2010). However, conflicting results showed that GNMs had positive effects or no obvious toxic effects. For example, Chen et al. found that GO sheets enhanced the growth and proliferation of a set of gut microorganisms by acting as a membrane scaffold (Chen et al., 2014). Ruiz et al. found that GO can be a general enhancer of cell growth for both bacterial and mammalian cells rather than an intrinsic antibacterial material (Ruiz et al., 2011). Use of different culture media has been considered to be one reason that lead to the contradictory antibacterial activities of GNMs (Hui et al., 2014). Hui et al. suggested that the adsorption of the organic substances in rich medium on GNMs might reduce the availability of the basal planes of GNMs, which may result in a lower antibacterial activity of GNMs (Hui et al., 2014).

The above studies deepened our understanding of the interaction between GNMs and bacteria. However, impact of GNMs on microbes from an environmental aspect is not well understood. In natural environment, the majority of the bacteria tend to colonize abiotic and biotic surfaces by forming sessile microbial consortia called biofilm (O'Toole et al., 2000). Bacterial cells closely attach to the substrate surface and adhere to each other through excreting EPS, and form biofilm with a highly hydrated and gel-like structure (O'Toole et al., 2000; Flemming et al., 2007). Evidence has shown that biofilm was potentially a main accumulator of environmental NMs in aquatic ecosystem (Ferry et al., 2009). Due to the natural barrier of EPS, biofilm can be 10 to 10,000 times less susceptible to external stress (e.g., antibacterial drugs) than the planktonic bacteria (Olson et al., 2002). Previous studies also suggested that biofilms at different growth stages may show different sensitivity to toxic substances (Stewart and Costerton, 2001; Rodrigues and Elimelech, 2010). However, most of current studies were performed on planktonic bacteria in a short-term exposure; only few studies on the impact and behavior of GNMs in biofilm were reported, and most of them focus on fabricating graphene-based materials for antibacterial application (Ruiz et al., 2011; Carpio et al., 2012; Kumar et al., 2015). Ruiz et al. (2011) reported that bacteria can form dense biofilm in the presence of GO, indicating that GO was lack of antibacterial activity. In another study, $1000 \mathrm{mg} / \mathrm{L}$ of GO suspension significantly inhibited biofilm formation of four types of bacterial strains (Carpio et al., 2012). Biofilm formation on indium tin oxide surface was also inhibited after GO coating. To comprehensively evaluate the environmental impact of GNMs, more studies on the potential effects of GNMs on microbial biofilm are required.

In natural environmental conditions, GNMs may interact with bacteria in different scenarios. On one hand, GNMs may contact with planktonic bacteria cells and influence the cell growth, adhesion and subsequent biofilm formation. On the other hand, GNMs may directly influence mature biofilm that have been attached on a surface (e.g., rocks, plant leaves) and affect the biofilm development (e.g., induce detachment of cells from biofilm). Evidence has shown that ENMs impacted biofilm differently in the two scenarios. Rodrigues and Elimelech
(2010) found that single-walled carbon nanotubes (SWCNTs) strongly inhibited the cell growth and biofilm formation when contacting with planktonic bacterial cells prior to the biofilm formation, while showed lower toxicity to the mature biofilm. We speculated that bacteria in the two scenarios may also respond differently to GNMs. Furthermore, due to the presence of large amount of EPS excreted by biofilm, response of biofilm to GNMs may be also different from planktonic bacteria in suspension which were used in many recent studies.

Most of the antibacterial test of GNMs in current literatures were carried out in saline or minimal medium solution; however, toxicity and behavior of GNMs in rich medium are not well understood yet. Since culture medium is an important factor affecting the antibacterial properties of GNMs, studies in different culture media are necessary to fully understand the toxicity and behavior of GNMs in environment. Moreover, the scenario of bacteria in rich medium do exist, e.g. in urban wastewater (Órpez et al., 2009). In the present study, we compared the impacts of GO and rGO on the biofilm formation and development of E. coli and S. aureus. Specifically, we investigated: 1) the impact of GO and rGO on total cell growth and biofilm formation, 2) effects of GO and rGO on the developed biofilms, 3) how bacteria affect the physicochemical properties of GO and rGO, and 4) the mechanism involved in the interaction of GNMs with bacteria. This study will provide significant insights into the impact of GNMs on microorganisms and mechanistic understanding of the toxicity and behavior of GNMs in bacterial biofilm.

\section{Materials and methods}

\subsection{Characterization of $G O$ and $r G O$}

GO and rGO were purchased from Chengdu Organic Chemicals Co. Ltd. (Chengdu, China). The carbon related chemical species were determined by XPS (ESCALAB 250Xi, Thermo Scientific, USA) equipped with a twin-crystal monochromatic X-ray source. Raman spectra were measured using a LabRAM HR Evolution Raman spectrometer (Horiba Scientific, Japan). FTIR spectra were measured with a Bruker Tensor 27 spectrometer. UV-vis spectra of GO and rGO suspension (100 mg/L) were recorded using a UV-vis spectrophotometer (Purkinje General, Beijing).

\subsection{Bacterial strains and culture}

The E. coli (Pbr 322) and S. aureus (ATCC 25923) were provided by Institute of Microbiology, Chinese Academy of Sciences. Cells were grown on LB (Luria-Bertani) agar plates for $24 \mathrm{~h}$ at $37^{\circ} \mathrm{C}$. A single colony was picked and inoculated in LB medium and incubated overnight at $37^{\circ} \mathrm{C}$ under $250 \mathrm{rpm}$ shaking speed. The cultures were harvested in the mid-exponential growth phase by centrifugation at $6000 \mathrm{rpm}$ for $10 \mathrm{~min}$. The pellets were resuspended in LB medium to obtain a final cell suspensions $\left(\mathrm{OD}_{600}=0.5\right.$ for the following experiments).

\subsection{Total cell growth and biofilm formation in GO and rGO suspensions}

A serial concentrations of GO and $\mathrm{rGO}(0,0.5,5,10,50,100,250$ and $500 \mathrm{mg} / \mathrm{L}$ ) suspensions were prepared in sterilized LB medium. Each concentration of GO or rGO suspensions was added to 12 wells of the sterilized 96-well flat-bottom microtiter plates with each well $300 \mu \mathrm{L}$, and then inoculated $3 \mu \mathrm{L}$ diluted $E$. coli and S. aureus suspensions in 6 of the 12 wells, with the other 6 wells as bacteria-free blanks. All the plates were incubated statically for $48 \mathrm{~h}$ at $37^{\circ} \mathrm{C}$. Total cell growth which represents the cells in the supernatant, cell aggregates on bottom, and the biofilm attached to the bottom was measured by recording the $\mathrm{OD}_{600}$ under a microplate reader (Infinite 200 Pro, Tecan, Switzerland). The readings from blank groups were averaged out and subtracted from the readings of wells with bacteria. After the $\mathrm{OD}_{600}$ measurement, $\mathrm{GO}$ and rGO were collected from wells by centrifugation, and heated with 
lauryl sodium sulphate solution to digest the bacterial cells (Liu et al., 2015). Salts and organic components were removed from the materials by washing with hydrochloric acid and ethanol repeatedly (Liu et al., 2015). The obtained materials were washed with deionized water and freeze-dried for XPS analyses (ESCALAB 250Xi, Thermo Scientific, USA). For SEM analyses, bacteria treated with $250 \mathrm{mg} / \mathrm{L} \mathrm{GO}$ and $\mathrm{rGO}$ were collected at $48 \mathrm{~h}$, washed and fixed with $2.5 \%$ glutaraldehyde for $1 \mathrm{~h}$ and dehydrated with gradient concentrations of ethanol. A drop of the dehydrated suspension was applied on a silica wafer and freezedried for SEM observation (S4800, Hitachi, Japan).

Biofilm formation was quantified by a crystal violet staining method (Djordjevic et al., 2002). Briefly, after measurement of total cell growth, the suspensions in the wells were removed and the wells were rinsed with PBS buffer ( $10 \mathrm{mM}, \mathrm{pH} 7.4$ ) three times to remove the suspended and unbound cells. The remaining biofilm on the walls were stained with $250 \mu \mathrm{L} 0.1 \%$ crystal violet solution for $20 \mathrm{~min}$. The solutions were then discarded and the dye in stained biofilm were released by adding $300 \mu \mathrm{L}$ acetone/ethanol (1:4,v/v), and the absorbance at $540 \mathrm{~nm}$ $\left(\mathrm{OD}_{540}\right)$ were recorded under a microplate reader. Readings from blanks were averaged out and subtracted from that containing bacteria.

Biofilm formation was also observed on a confocal laser scanning microscopy (CLSM). Bacteria suspensions were inoculated in $500 \mathrm{mg} / \mathrm{L} \mathrm{GO}$ and rGO suspensions $(1: 100, v / v)$ in a six well microplate embedded with sterilized coverslip on the bottom and incubated at $37{ }^{\circ} \mathrm{C}$ for $48 \mathrm{~h}$. The coverslips were then rinsed with PBS for three times and stained with the LIVE/DEAD BacLight Bacterial Viability Kit (Invitrogen Corp., Carlsbad, CA) according to the provided protocol. Propidium iodide (PI) and SYTO9 in the kit were used for probing dead and live cells respectively. Images of the biofilm on the coverslip were taken on a CLSM (TCS SP5, Leica, Germany). Stack images were obtained by scanning the slides along the $Z$-axis at $2 \mu \mathrm{m}$ intervals down through the biofilm. The images were then analyzed in COMSTAT to calculate the total biomass, area occupied by biofilm, the average and maximum thickness, and the roughness coefficient of the biofilm (Heydorn et al., 2000).

ROS generation during the biofilm formation was determined by nitro blue tetrazolium (NBT) reduction assay (Gurunathan et al., 2012). The measurement of ROS contents related to graphene has been reported (Gurunathan et al., 2012; Qiu et al., 2014; Zhang et al., 2016). ROS contents in the suspensions and biofilm were measured at $6,12,36$, and $48 \mathrm{~h}$. Briefly, NBT $(1 \mathrm{mg} / \mathrm{mL})$ was added into the suspension or biofilm at different time points, and allowed to react at $37^{\circ} \mathrm{C}$ for $30 \mathrm{~min}$. The insoluble formazan were then extracted by $\mathrm{KOH}$ and DMSO $(v / v: 1: 3)$, and the absorbance at $630 \mathrm{~nm}$ was recorded under the microplate reader. It has been reported that GO or rGO may adsorb dyes and metals (Kemp et al., 2013); therefore, adsorption of NBT on GO and rGO were investigated to validate the feaibility of using NBT in this study. Results showed that the absorption of NBT at $260 \mathrm{~nm}$ were not significantly altered after incubation with GO and rGO (Fig. S1). Therefore, the effect of adsorption of NBT on the ROS could be neglected.

\subsection{Cell growth of planktonic bacteria in $\mathrm{GO}$ and $\mathrm{rGO}$ suspensions}

Effect of GO and rGO on the growth of planktonic bacteria were investigated by comparing the suspension assay with the stationary assay. Bacterial cultures obtained as described in the previous section were diluted by 100 -fold with GO and rGO suspensions $(0,5,10,50$, 100,250 and $500 \mathrm{mg} / \mathrm{L}$ ), and allowed to grow under shaking with a speed of $250 \mathrm{rpm}$ for $48 \mathrm{~h}$. $\mathrm{OD}_{600}$ was measured and subtracted from the bacteria-free blanks.

\subsection{Exposure of mature biofilm to GO and $r G O$ with or without supplement of soluble EPS}

E. coli or S. aureus were inoculated in $200 \mathrm{~mL}$ LB medium and incubated at $37^{\circ} \mathrm{C}$ statically for $48 \mathrm{~h}$. The suspensions were then filtered with a $0.22 \mu \mathrm{m}$ membrane (Millipore, USA) and dialyzed to remove the cells, nutrients and metabolites. The obtained filtrates were solutions containing large amount of soluble EPS. GO and rGO suspensions $(0.5,5,10,50,100,250$ and $500 \mathrm{mg} / \mathrm{L})$ were then prepared in EPS solution (named as "EPS +"). The same concentrations of GO and rGO were prepared in LB media for comparison (named as "EPS-"). Mature biofilm were obtained by incubating the bacteria in LB media in 96 microplates for $48 \mathrm{~h}$. The culture media were removed and the wells were rinsed with PBS (10 mM, pH 7.4) carefully. The attached biofilm in the wells were then incubated with the EPS + or EPS- GO and rGO suspensions at $37^{\circ} \mathrm{C}$. EPS solution and LB media with bacteria were set as controls. After incubation for $2 \mathrm{~h}$, suspensions were removed from the wells and the biofilm biomass were quantified by the crystal violet staining method as described above. ROS generation in the suspension (extracellular ROS, eROS) and biofilm (intracellular ROS, iROS) were measured according to the procedure described in the previous section.

\subsection{Statistical analysis}

All tests were repeated at least three times. Data were expressed as mean $\pm \mathrm{SD}$ (standard deviation). Statistical significance was analyzed by ANOVA or student's $t$-test. $P<0.05$ was considered as the level of significance.

\section{Results and discussion}

\subsection{Characterization of $\mathrm{GO}$ and $r G O$}

Physicochemical properties of GO and rGO were shown in Figs. S2S4. As shown in Fig. S2, FTIR spectra of GO showed abundant oxygen containing groups (e.g., $\mathrm{C}=\mathrm{O}$ at $1726 \mathrm{~cm}^{-1}, \mathrm{O}-\mathrm{H}$ at $3395 \mathrm{~cm}^{-1}$ and $1410 \mathrm{~cm}^{-1}$, and $\mathrm{C}-\mathrm{O}$ at $1226 \mathrm{~cm}^{-1}$ and $1052 \mathrm{~cm}^{-1}$ ) compared with that of rGO. UV-vis spectra of GO (Fig. S3) showed a typical adsorption at $230 \mathrm{~nm}$ which was presumably due to the excitation of $\pi$-plasmon of $\mathrm{C}-\mathrm{C}$ bonds. Absorption of rGO in the whole range was flat probably due to the black graphene-like structure. Raman spectra showed that the electronic conjugations of GO and rGO were different (Fig. S4). Intensity ratio of $D$ band at $1350 \mathrm{~cm}^{-1}$ to $G$ band at $1596 \mathrm{~cm}^{-1}$ were respectively 1.06 and 0.96 for $\mathrm{GO}$ and $\mathrm{rGO}$, indicating higher amount of defects in GO structure.

\subsection{Effects of GO and rGO on the total cell growth}

Fig. 1 showed the total cell growth of bacteria. GO and rGO showed completely different impacts on bacterial growth in LB media. GO showed concentration dependent enhancement of the total cell growth at all concentrations after $12 \mathrm{~h}$. In contrast, rGO showed significant toxicity on the two bacteria models. Cell growth was inhibited by rGO at concentrations from 50 to $250 \mathrm{mg} / \mathrm{L}$, and was retarded at $500 \mathrm{mg} / \mathrm{L}$. Interestingly, the inhibitory effects of 50 and $100 \mathrm{mg} / \mathrm{L}$ of rGO were attenuated at $24 \mathrm{~h}$, and eliminated at $48 \mathrm{~h}$. Studies have shown that the antibacterial effect of GNMs are subject to the influence of experimental surroundings such as culture media, $\mathrm{pH}$, light, and magnetic field etc. (Zou et al., 2016). GO in saline solution exhibited antibacterial properties; however, in nutrient broth GO usually showed low or no antibacterial activity, or even positive effect (Zou et al., 2016). Hui et al. suggested that noncovalent adsorption of nutrient components on GO basal planes might account for the deactivation of the antibacterial activity of GO (Hui et al., 2014). Such mechanism may also apply to this study. LB medium contains large amount of proteins, which may be adsorbed on GO. Adsorption of the proteins may not only deactivate the antibacterial property of GO, but also enhance the bacterial growth by providing sufficient nutrients on GO scaffold. In contrast, rGO in LB media exhibited significant antibacterial activity. A recent study showed that rGO possessed lower adsorption ability of proteins than GO (Wei et al., 2015), which may result in a lower deactivation of its antibacterial properties. 


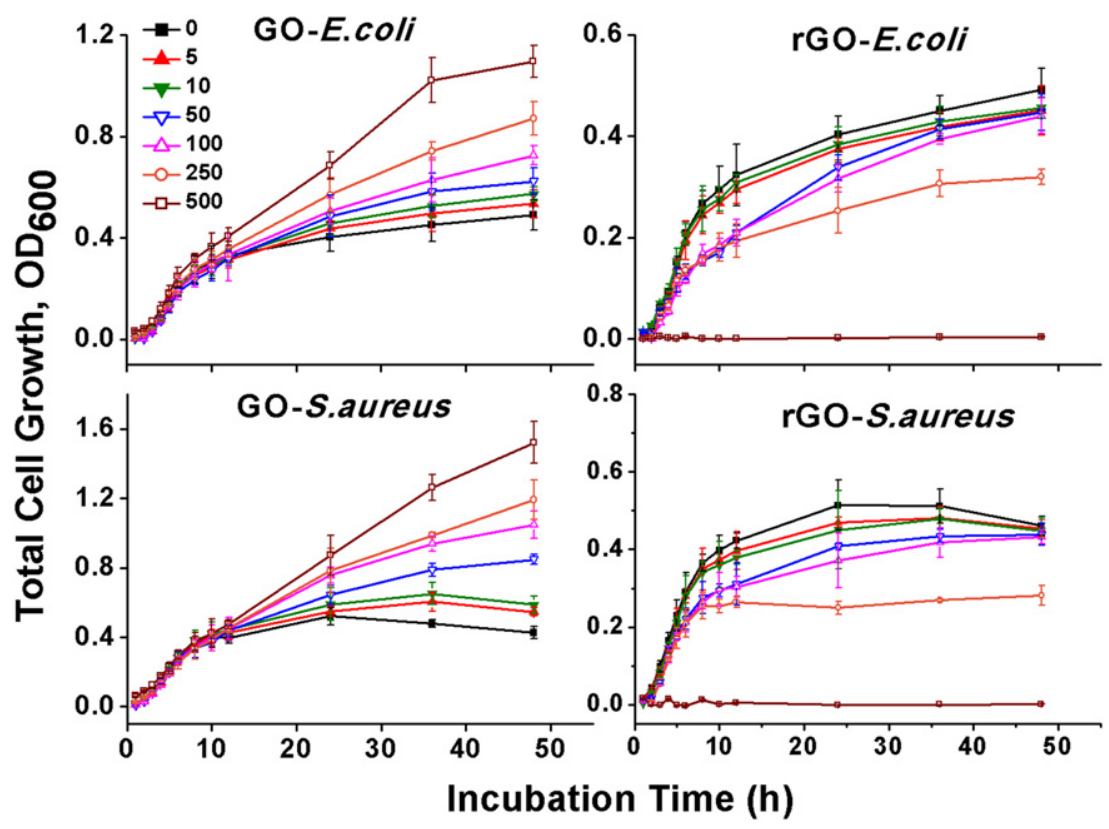

Fig. 1. Effect of GO and rGO on total cell growth of E. coli and S. aureus.

Indeed, we incubated GO and rGO with LB media, and found that GO adsorbed more LB components than rGO (Fig. S5), which may be an important reason for the discrepancy of their bacterial effect. Studies on the interaction of GO and rGO with various components in culture media are required.

Responses of planktonic bacteria to $\mathrm{GO}$ and rGO were also evaluated by a suspension assay (Fig. S6). GO promoted the growth of bacteria while rGO inhibited the growth of bacteria. Higher toxicity of rGO was found in suspension assays than in the stationary assays. Bacterial concentrations of $E$. coli and S. aureus were inhibited by $60.4 \%$ and $67.6 \%$, which were much higher than the values in stationary assay $(37.5 \%$ and $39.8 \%$, respectively). The toxicity of rGO (50 and $100 \mathrm{mg} / \mathrm{L}$ ) was also attenuated at $36 \mathrm{~h}$; however, the degree of attenuation was lower than that in stationary bacteria. The discrepancy between stationary and suspension assays may be attributed to two reasons: 1) bacteria at stationary condition may excrete large amount of EPS which can protect the bacteria during the biofilm formation; 2) shaking in suspension assay resulted in more chance of contact of rGO with bacteria. Both of the two factors may result in the different impact of rGO on the two different bacteria models.

Interestingly, this study found that the toxicity of rGO to bacteria was attenuated with the increasing of incubation time, indicating that bacteria showed resistance to rGO. Resistance of bacteria to antibiotics and drugs has been reported by numerous studies (Davies, 2003). For example, antibiotics including ampicillin, cefalexin, tetracycline and minocycline significantly inhibited biofilm formation of Gram-negative bacterium Actinobacillus actinomycetemcomitans in the early phase (0$24 \mathrm{~h}$ after inoculation), while the toxic effect decreased and finally disappeared in the mature phase (24-48 h after inoculation) (Takahashi et al., 2007). We proposed that the resistance of bacteria to rGO may share similar mechanisms with that for antibiotics, in particular, EPS may play an important role as a natural barrier for protection (Davies, 2003). In addition, we proposed that the "resistance" may not only originate from the bacteria itself, but also be related to the transformation of rGO. These hypotheses were verified and discussed in the following sections. It should be noted that the toxicity of higher concentrations of rGO ( 250 and $500 \mathrm{mg} / \mathrm{L}$ ) were not attenuated; this was probably due to the reason that most of the cells initially inoculated in the media were inactivated by the high concentrations of rGO.

\subsection{Effects of $G O$ and $r G O$ on biofilm formation}

Effects of GO and rGO exposure on biofilm formation were evaluated by a crystal violet staining method. GO enhanced the biofilm formation of both bacteria models at concentrations from 50 to $500 \mathrm{mg} / \mathrm{L}$ (Fig. 2). Biofilm biomasses were reduced when exposed to high concentrations of rGO while there were no significant effects at concentrations ranging from 0 to $50 \mathrm{mg} / \mathrm{L}$. To further intuitively understand the effect of GO and rGO on the biofilm formation, we observed the biofilm using a Live/ Dead molecular probe assay. As shown in Fig. 3, more live cells were observed for GO treatment when compared with control, while only a

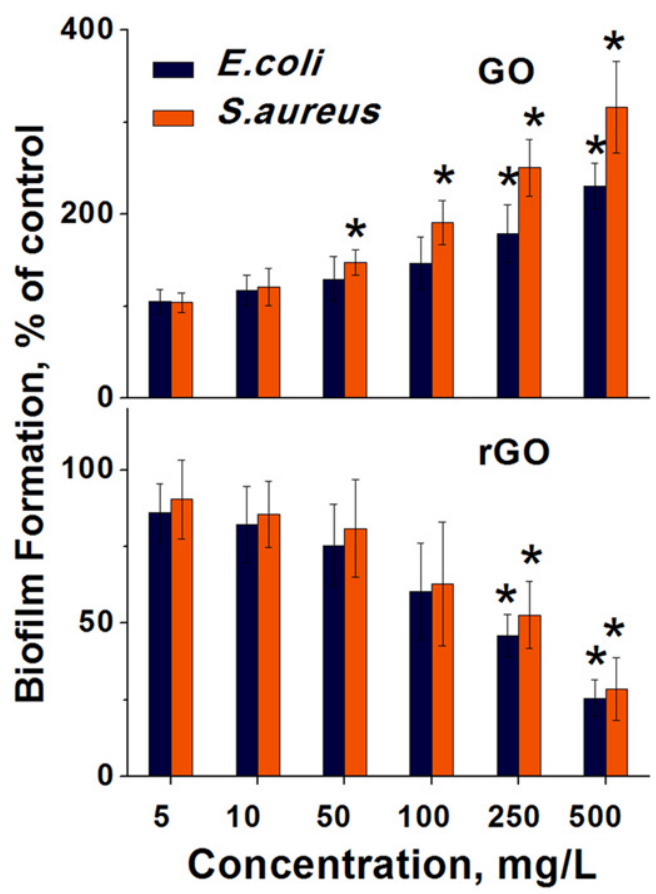

Fig. 2. Biofilm formation after incubation with GO and rGO for $48 \mathrm{~h}$. * indicates significant difference at $P<0.05$ compared with control. 


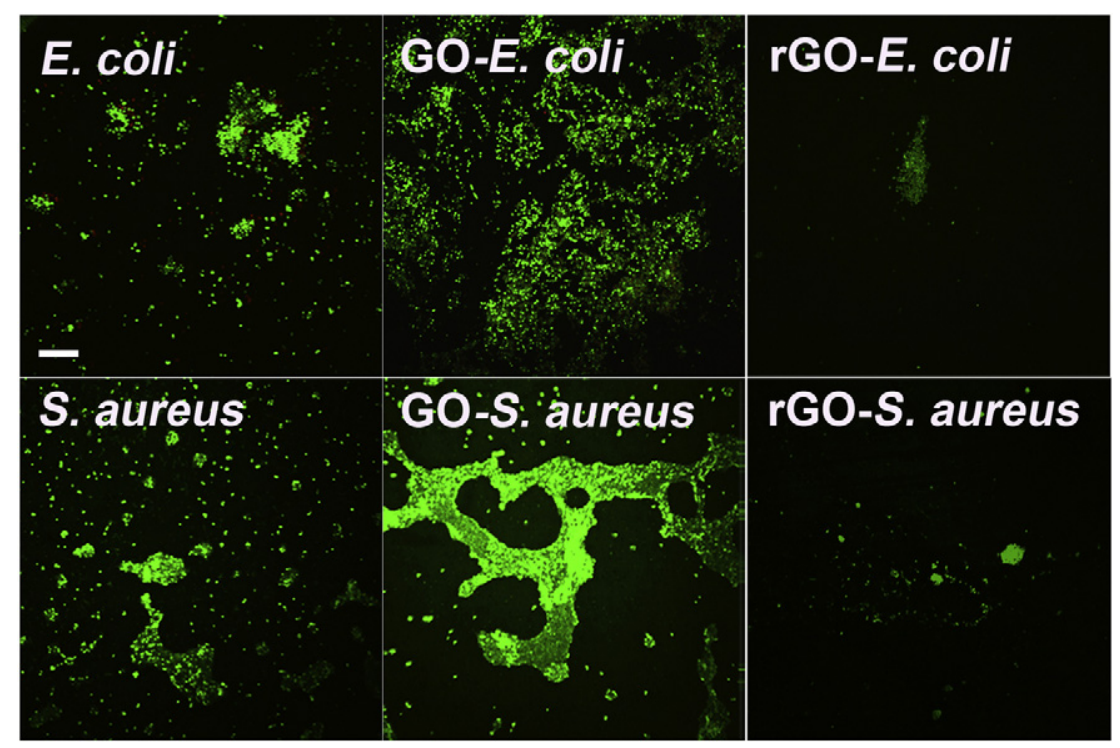

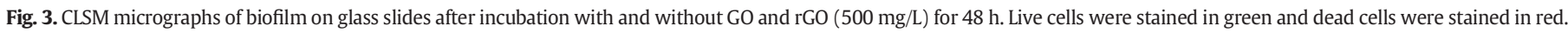
Images were obtained by overlaying the red and green colors. White bar in the images indicates $100 \mu \mathrm{m}$.

small amount of live cells were observed for the rGO treatment. Different colony patterns of $E$. coli and S. aureus were observed. Biofilm of $S$. aureus was more dense but with a smaller coverage area than E. coli. Quantitative information of the biofilm structure (Table 1) was obtained by analyzing the stack images using a three-dimensional biofilm program COMSTAT. GO significantly increased the total biomass and substratum coverage by $124 \%$ and $280 \%$ for E. coli, and $102 \%$ and $126 \%$ for S. aureus. Average and maximum thickness also increased. These results indicated that GO promoted the colony of cells on the substrate and enhanced the biofilm growth. The roughness coefficient was not altered by $\mathrm{GO}$, indicating the biofilm grew uniformly. In contrast, all parameters were significantly reduced by rGO except for the roughness coefficient, indicating that cells can hardly colonize on the substrate.

\subsection{Effect of GO and rGO on developed biofilms and role of EPS}

Impact of GO and $\mathrm{rGO}$ on the growth of mature biofilm and the role of EPS in the impact were investigated in this section. As shown in Fig. 4, GO showed concentration dependent increase of biofilm biomass. rGO at high concentrations ( $\geq 100 \mathrm{mg} / \mathrm{L}$ for $E$. coli and $\geq 250 \mathrm{mg} / \mathrm{L}$ for $S$. aureus) inhibited the biofilm growth (Fig. 4B and D); however, the inhibitory effect was lower compared with that during the biofilm formation (Fig. 2), indicating that mature biofilm was more resistant to rGO. This might be attributed to the presence of EPS. In mature phase, almost $75-95 \%$ of the volume of biofilm is occupied by EPS, which is primarily composed of polysaccharides, proteins, and nucleic acids. It forms a hydrated gel-like structure which can stabilize the bacteria cells and enable the communication between the cells (Flemming et al., 2007), and plays a significant role in protecting the bacteria against ambient threat (e.g. extreme temperature, dehydration, antibiotics, heavy metals) (Olson et al., 2002). Indeed, our results showed that supplement of extracted EPS mitigated the toxic effect of rGO (Fig. 4B and D), indicating that EPS played an important role as a barrier against rGO. The negatively-charged EPS may surround the positively-charged rGO (Zeta potential: $10.6 \pm 2.4 \mathrm{mV}$ ) and reduce the transport and penetration of rGO, which may prevent the bacteria from contacting with rGO and mitigate the toxicity. Quenching of ROS by EPS might be another mechanism of EPS protection (Diao et al., 2014). These results demonstrated our hypothesis that EPS may contribute to the attenuation of the toxicity of rGO in the mature phase of biofilm formation. After entering into mature phase, more EPS were excreted by the bacteria, acting as a natural barrier and ROS sink. Interestingly, the positive effects of GO were slightly enhanced by the presence of EPS. Biofilm develops via adherence of bacteria cells and colonization on substrate through EPS. Given that GO could adsorb LB components (Fig. S5), it may also adsorb the components of EPS, thus preconcentrate the extra EPS on GO sheet and facilitate the combination of cells and colonization of bacteria and the growth of biofilm. Further studies are needed to investigate the interaction of GO or rGO with EPS in the future.

\subsection{Biotransformation of $G O$ and $r G O$}

Physicochemical properties of ENMs determine their fate and toxicity in environment. Interaction with bacteria may modify the physicochemical properties of GNMs and determine their behavior and toxicity. To further understand the interaction of GNMs with bacteria,

Table 1

Structure properties of E. coli and S. aureus biofilm after incubation with and without the presence of GO and rGO (500 mg/L) for 48 h obtained by CLSM. ${ }^{\text {a }}$

\begin{tabular}{|c|c|c|c|c|c|c|}
\hline Analyses $^{\mathrm{a}}$ & E. coli & GO-E. coli & rGO-E. coli & S. aureus & GO-S. aureus & rGO-S. aureus \\
\hline Total biomass $\left(\mu \mathrm{m}^{3} / \mu \mathrm{m}^{2}\right)$ & $2.0 \pm 0.8$ & $6.8 \pm 3.7^{*}$ & $0.4 \pm 0.2^{*}$ & $3.1 \pm 0.7$ & $13.9 \pm 2.0^{*}$ & $0.8 \pm 0.3^{*}$ \\
\hline Substratum coverage (\%) & $13.4 \pm 4.2$ & $27.1 \pm 4.4^{*}$ & $2.3 \pm 0.9^{*}$ & $9.4 \pm 3.2$ & $21.3 \pm 4.9^{*}$ & $2.5 \pm 0.7^{*}$ \\
\hline Average thickness $(\mu \mathrm{m})$ & $10.6 \pm 2.1$ & $15.7 \pm 4.9$ & $1.5 \pm 2.4^{*}$ & $12.6 \pm 2.1$ & $37.8 \pm 5.9^{*}$ & $3.8 \pm 2.2^{*}$ \\
\hline Maximum thickness ( $\mu \mathrm{m})$ & $16.0 \pm 2.3$ & $22.6 \pm 3.9^{*}$ & $3.8 \pm 2.0^{*}$ & $22.0 \pm 2.3$ & $43.3 \pm 8.3^{*}$ & $5.6 \pm 3.4^{*}$ \\
\hline Roughness coefficient & $1.2 \pm 0.1$ & $1.1 \pm 0.1$ & $1.2 \pm 0.1$ & $1.2 \pm 0.1$ & $1.1 \pm 0.1$ & $1.3 \pm 0.1$ \\
\hline
\end{tabular}

a Results were obtained from average of at least four slides for each condition. Analyses were performed on a COMSTAT program. Total biomass represents the overall volume per area

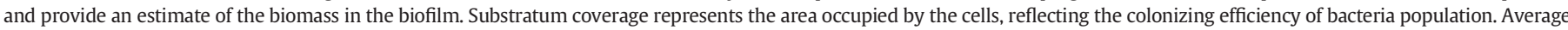

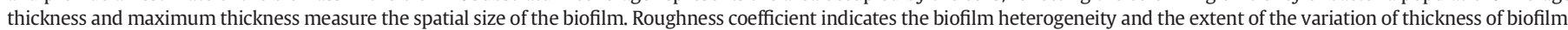

* Indicated a significant difference $(P<0.05)$ compared with control. 


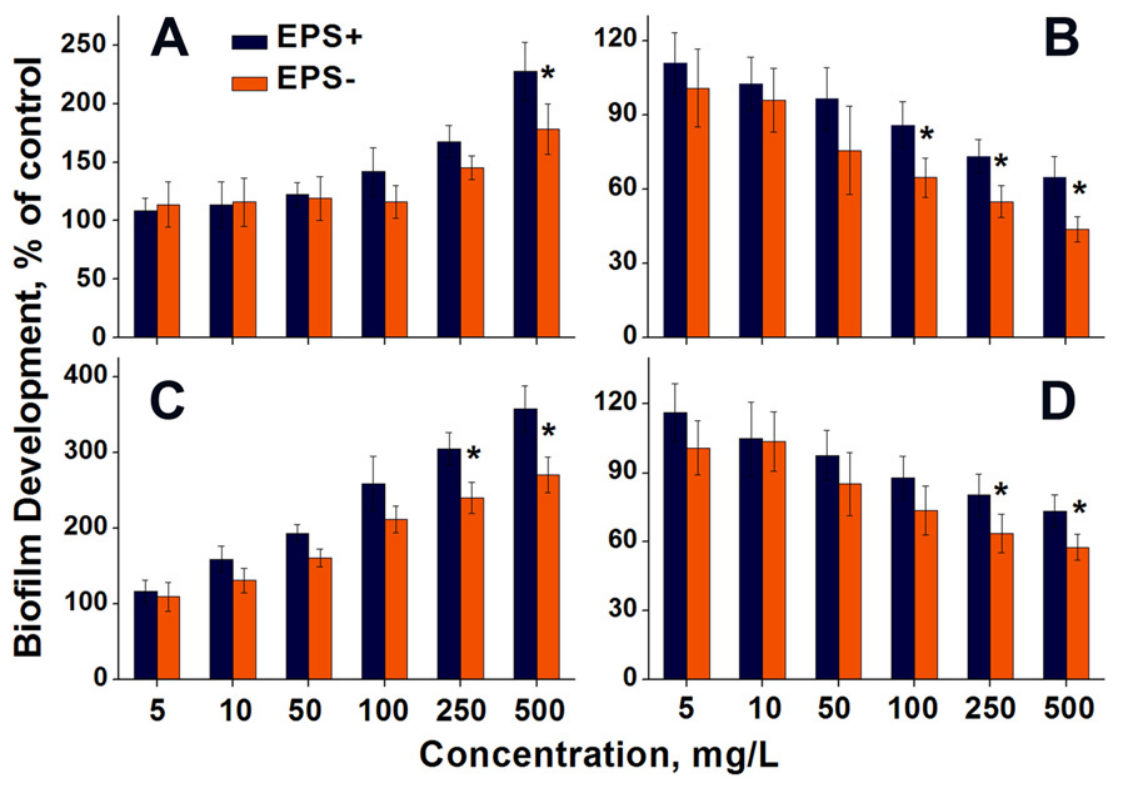

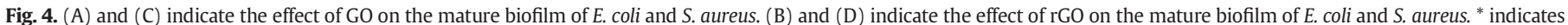
significant difference $(P<0.05)$ between group of EPS + and EPS-.

GO and rGO after incubation with bacteria were characterized by XPS. Fig. S7 shows the survey spectra of the samples. No tracing of $\mathrm{K}_{2} \mathrm{p}_{3 / 2}$ $(292.7 \mathrm{eV})$ corresponding to the $\mathrm{K}$ elements in the bacteria indicated that there was no interference of $C$ signal from the bacteria in these samples (Akhavan and Ghaderi, 2012). Fitting of the XPS spectra yielded typical bands centered at $284.8 \mathrm{eV}, 286.5 \mathrm{eV}, 287.9 \mathrm{eV}$, and $289.0 \mathrm{eV}$ (Fig. 5 ), which were respectively ascribed to $\mathrm{C}-\mathrm{C} / \mathrm{C}=\mathrm{C} / \mathrm{C}-\mathrm{H}, \mathrm{C}-\mathrm{O}$ (epoxy and alkoxy), $\mathrm{C}=\mathrm{O}$, and $\mathrm{O}=\mathrm{C}-\mathrm{OH}$ functional groups. After incubation with bacteria, the peak intensities of $\mathrm{C}-\mathrm{O}$ decreased significantly compared with the pristine GO, revealing the reduction of GO. Interestingly, rGO were oxidized after incubation with bacteria, indicated by the increase of the peak intensities of $\mathrm{C}-\mathrm{O}$. Relative contents of the functional groups were calculated by comparing the peak area ratios (Table 2).

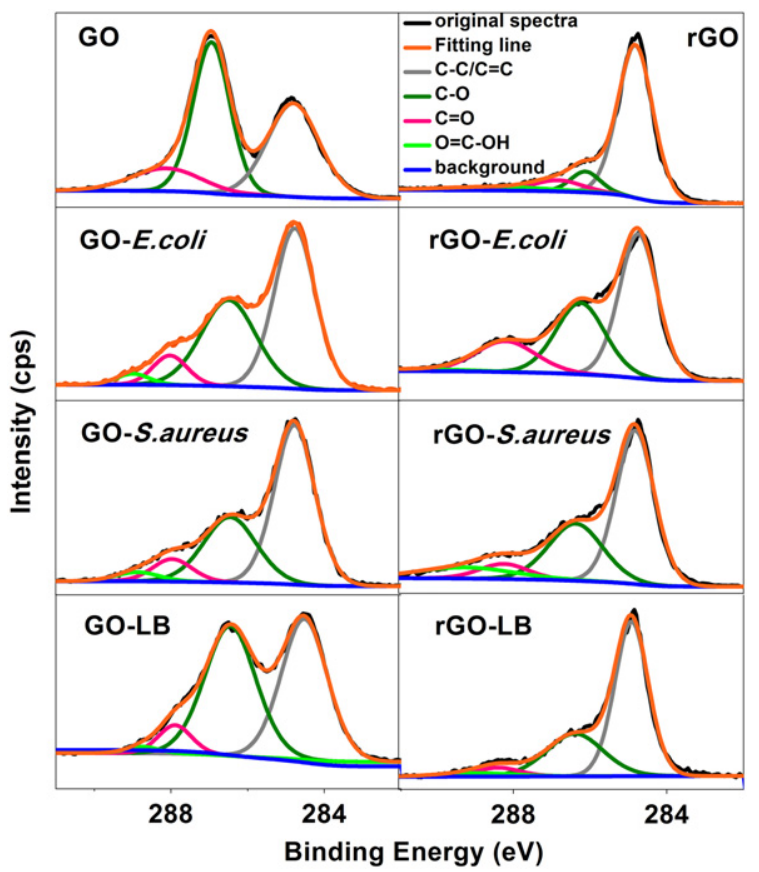

Fig. 5. XPS spectra of pristine GO and rGO, and GO or rGO after $48 \mathrm{~h}$ incubation with bacteria or LB media.
Percentages of $\mathrm{C}-\mathrm{C} / \mathrm{C}=\mathrm{C}$ in $\mathrm{GO}$ increased from $40.6 \%$ to $51.9 \%$ after incubation with $E$. coli and to $57.6 \%$ after incubation with $S$. aureus. In terms of $\mathrm{rGO}$, percentages of $\mathrm{C}-\mathrm{C} / \mathrm{C}=\mathrm{C}$ decreased from $77.4 \%$ to $49.7 \%$ and $51.4 \%$ after incubation with $E$. coli and S. aureus, respectively.

In order to determine if the LB medium was involved in the redox reaction, GO and rGO were incubated in LB medium for $48 \mathrm{~h}$ and characterized by XPS (Fig. 5). Percentage of oxygen-containing groups in GO was $41.5 \%$, which was not significantly different from that in the pristine GO (40.6\%), indicating that LB could not reduce GO and the reduction of GO we observed was induced by the interaction with bacteria. Percentage of oxygen-containing groups in rGO increased from $22.6 \%$ to $40.7 \%$ after incubation in LB media; however, the percentage was lower compared with rGO that was incubated with bacteria (50.3\% for E. coli and $48.6 \%$ for $S$. aureus), indicating that both LB and bacteria were involved in the oxidation of rGO.

Reduction of GO by bacteria has been reported in several studies. Salas et al. (2010) found that GO could be reduced by a series of Shewanella bacteria strains via the bacterial respiration, and the reduction process were mediated by the cytochromes. Reduction of GO by E. coli was also reported and was considered to be accomplished through the glycolysis process (Akhavan and Ghaderi, 2012). However, oxidation of rGO by bacteria were rarely reported. A recent study showed that rGO and graphite can be oxidized by a naphthalenedegrading Pseudomonas bacteria (Liu et al., 2015). Oxidation occurred at the defects and edges of the surface with higher reaction activity (Liu et al., 2015). In this study, rGO was oxidized to GO, which can enhance the bacteria growth in LB media, might be another possible reason for the attenuation of the toxicity of rGO.

Table 2

Fraction of carbon related functional groups in $\mathrm{GO}$ and $\mathrm{rGO}$ before and after incubation with bacteria or LB media for $48 \mathrm{~h}$.

\begin{tabular}{lllll}
\hline & $\mathrm{C}-\mathrm{C} / \mathrm{C}=\mathrm{C}(\%)$ & $\mathrm{C}-\mathrm{O}(\%)$ & $\mathrm{C}=\mathrm{O}(\%)$ & $\mathrm{O}=\mathrm{C}-\mathrm{OH}(\%)$ \\
\hline GO & 40.62 & 46.09 & 13.29 & - \\
GO-E. coli & 51.9 & 37.06 & 8.41 & 2.62 \\
GO-S. aureus & 57.59 & 30.85 & 7.99 & 3.57 \\
GO-LB & 41.53 & 48.93 & 6.20 & 1.34 \\
rGO & 77.41 & 8.90 & 7.91 & 5.78 \\
rGO-E. coli & 49.68 & 31.6 & 16.75 & 1.97 \\
rGO-S. aureus & 51.36 & 28.68 & 10.24 & 9.72 \\
rGO-LB & 69.28 & 21.88 & 4.15 & 4.70
\end{tabular}




\subsection{Physical interaction and oxidative stress mediated toxicity of rGO}

Antibacterial activities of GNMs have been proposed to be mediated by several mechanisms. Direct contact of bacteria with GNMs may cause physical damage of the cell membrane. Graphene with atomically sharp edges can penetrate the cell membrane and disrupt the membrane integrity (Akhavan and Ghaderi, 2010; Hu et al., 2010). A recent study reported that graphene and GO induced the degradation of E. coli cell membrane by penetrating and extracting large amount of phospholipids from cell membranes (Tu et al., 2013). Wrapping of bacteria by graphene sheets was also proposed to be one possible mechanism (Carpio et al., 2012; Akhavan et al., 2011). The bacterial cells which were trapped in the GO sheets were not able to proliferate in the culture medium and consume required nutrients from the surrounding environment. Another study compared the antibacterial activity of GO-Ag and GO sheets. However, results showed that GO sheets showed no toxicity even though it attached and wrapped bacteria, indicating that the antibacterial activity did not simply originate from the wrapping (Ocsoy et al., 2013). In our study, most of the E. coli (Fig. 6A1 and A2) and S. aureus cells (Fig. 6B1 and B2) were covered or wrapped in the GO sheets; however, the wrapping or trapping did not induce any toxicity. Instead, our results were in accordance with several previous reports that GO was not toxic and could be a non-specific enhancer of cellular growth including bacteria (Ruiz et al., 2011). GO may provide a preconcentration platform of nutrients for bacterial growth (Chen et al., 2014; Ruiz et al., 2011). No wrapping were observed for rGO in this study. Instead, bacteria cells closely contacted with the lateral edges of rGO, indicating that physical damage of the cell membrane may be one possible reason of rGO toxicity to bacteria.

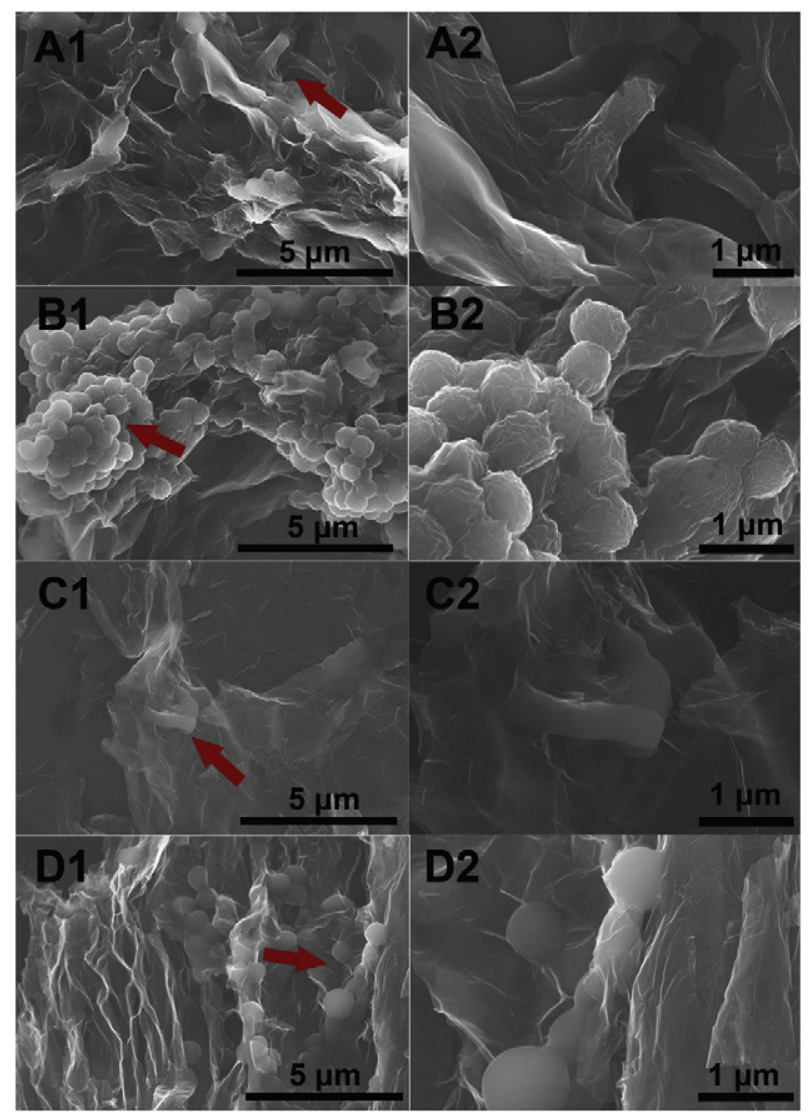

Fig. 6. SEM images of bacteria after incubation with GO and rGO for $48 \mathrm{~h}$. (A) and (C) indicate E. coli incubated with GO and rGO. (B) and (D) indicate S. aureus incubated with GO and rGO. A2-D2 indicate the magnifications of the regions in A1-D1 as shown by the red arrows.
In addition to the physical damage, oxidative stress was considered to be a major mechanism involved in the antibacterial activity of GNMs (Djordjevic et al., 2002; Krishnamoorthy et al., 2012). It has been reported that GO and rGO could lead to oxidative stress either through the direct formation of reactive oxygen species or through the depletion of cellular antioxidants (e.g., glutathione) (Liu et al., 2011; Perreault et al., 2015). Pre-incubation of bacterial cells with glutathione can reduce the antibacterial activity of GO nanosheets (Perreault et al. 2015). Oxidative nature of GO could induce membrane damage through lipid oxidation, which was also reported for carbon nanotubes and fullerene (Lyon and Alvarez, 2008; Vecitis et al., 2010). To further understand the discrepancy in the toxicity of GO and rGO in this study, ROS generation was examined at both biofilm formation and mature biofilm stages. As shown in Fig. 7A and Fig. 8, GO showed no significant effect on ROS levels at concentrations from 5 to $100 \mathrm{mg} / \mathrm{L}$ while reduced the ROS contents at $250 \mathrm{mg} / \mathrm{L}$. In contrast, rGO treatment enhanced the ROS generation. During biofilm formation, ROS contents in biofilm significantly increased after treatment with $\mathrm{rGO}(\geq 50 \mathrm{mg} / \mathrm{L})$ for $12 \mathrm{~h}$, while decreased to a similar level as control at $48 \mathrm{~h}$ (Fig. 7B). Increase of ROS content and physical interaction may induce membrane damage of the cells (Kamat et al., 2000). Indeed, we examined the membrane damage using the Live/dead probe. Results showed that rGO (500 mg/L) induced significant membrane damage to the developed biofilm after exposure for $2 \mathrm{~h}$ (Fig. S8).

Cells in suspensions were more sensitive than biofilm, where ROS levels were increased by rGO ( $\geq 100 \mathrm{mg} / \mathrm{L}$ ) at $6 \mathrm{~h}$; however, ROS contents decreased to a similar level as control at $48 \mathrm{~h}$ except for that in $250 \mathrm{mg} / \mathrm{L}$ treatment. These findings suggested that toxicity of rGO to bacteria was mediated by the oxidative stress. These findings were also correlated well with the impact of GO and rGO on the total cell growth and biofilm formation. It has been reported that EPS could protect the bacteria from antimicrobials including ROS by acting as a reactive sink (Starkey et al., 2004). It could be deduced that decrease of ROS levels at mature phase might be attributed to the excretion of EPS during the biofilm development. Quenching of ROS by EPS may be an important reason for the attenuation of the toxicity of rGO.

We further examined the ROS generation in developed biofilm (Fig. 8). Intracellular ROS (iROS) contents increased only in $250 \mathrm{mg} / \mathrm{L}$ rGO treatment, while the extracellular ROS (eROS) levels in the biofilm were not altered by rGO. This was because mature biofilm was abundant with EPS, which may protect the biofilm by quenching the ROS in the EPS matrix. Taken together, the antibacterial property of rGO was induced by both physical damage and oxidative stress. EPS could not only act as a barrier to prevent physical damage caused by rGO, but also act as a sink of ROS, thereby protecting the bacteria from the toxicity of rGO.

\section{Conclusion}

The present study showed that GO and rGO exhibited completely different effects on bacteria growth, biofilm formation and development in LB media. Both physical interaction and oxidative stress were involved in the toxicity of rGO. Responses of bacteria to GNMs at different growth stages were different. Toxicity of rGO was attenuated with the formation and development of biofilm. EPS, which could act as a natural barrier and a sink of ROS, played a critical role in the attenuation of the toxicity of rGO. Oxidation of rGO to GO, which can enhance the bacterial growth and biofilm formation, might be another reason for the attenuation of the toxicity of rGO. This study implies that effect of GNMs on biofilm should be evaluated to fully understand the impact of these compounds on bacteria. It should be noted that this study was carried out in rich media, impact of GNMs on biofilm in a minimal media should be also evaluated in the future.

\section{Notes}

The authors declare no competing financial interest. 

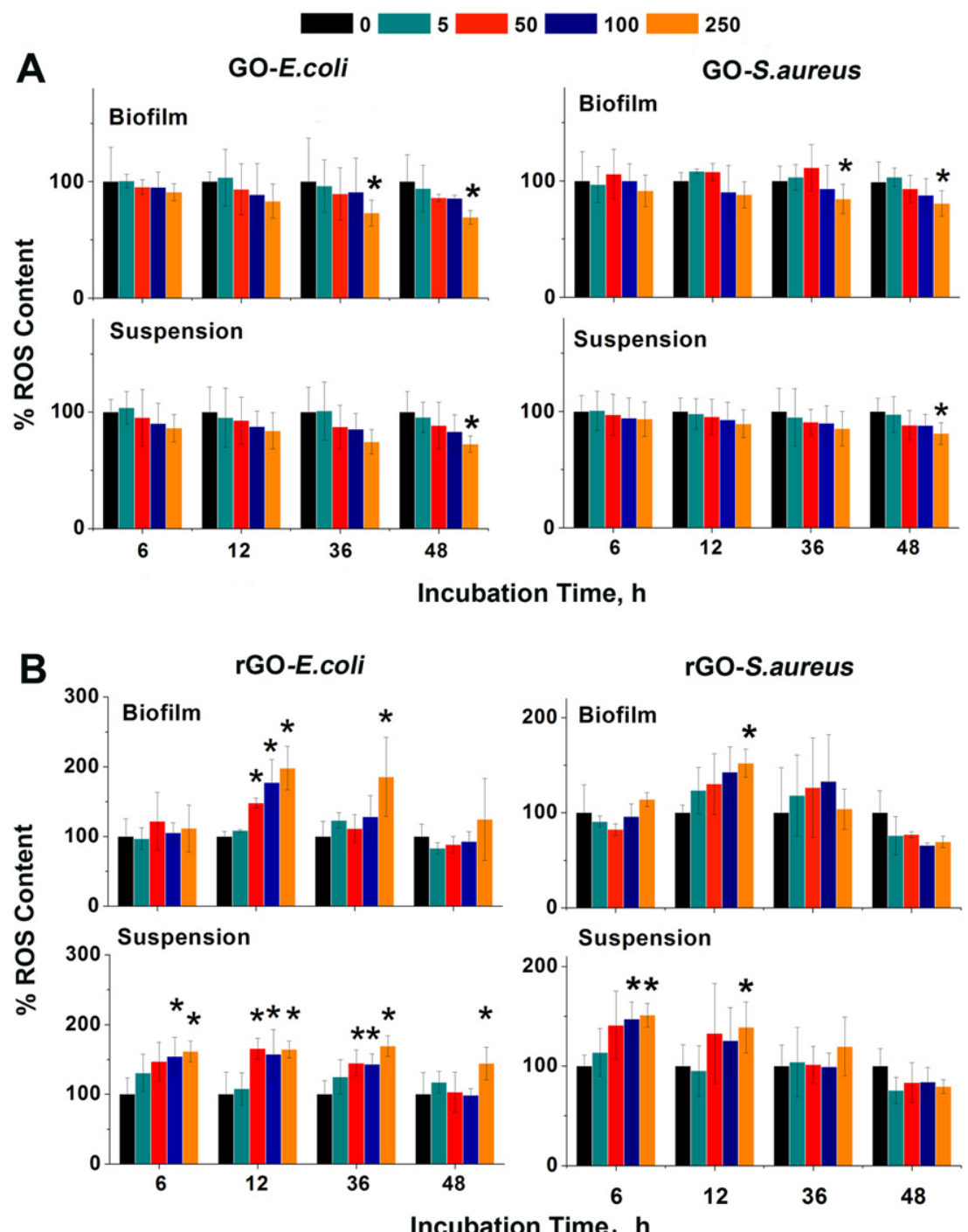

Fig. 7. ROS generation in biofilm and suspension after incubation with GO (A) and rGO (B) for 6, 12, 36 and 48 h. ${ }^{*}$ indicates significant difference $(P<0.05)$ compared with control.

\section{Acknowledgement}

This work was financially supported by the National Natural Science Foundation of China (Grant No. 11405183, 11275215, 11275218,

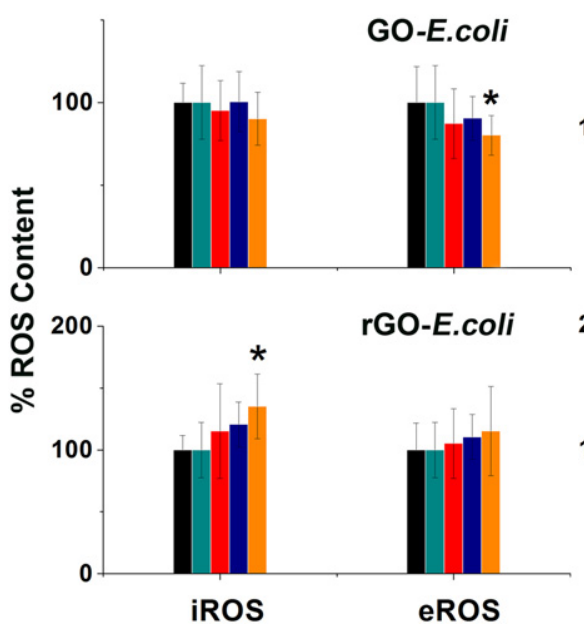

$21507153,11375009,11575208)$, Ministry of Science and Technology of China (Grant No. 2011CB933400, 2013CB932703), and Youth Innovation Fund of Institute of High Energy Physics, Chinese Academy of Sciences (Grant No. Y454631).
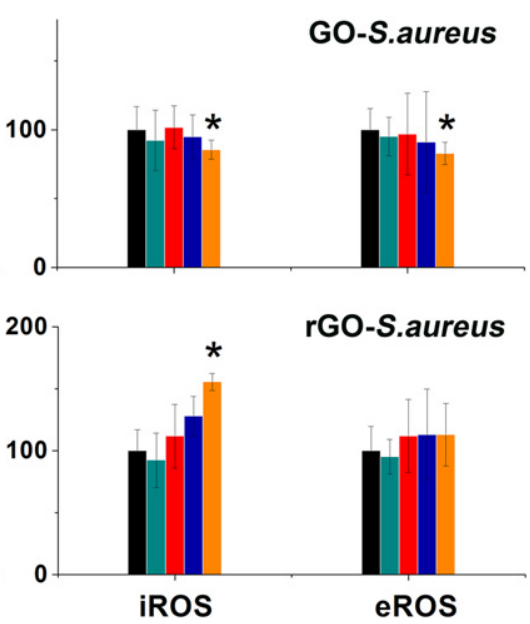

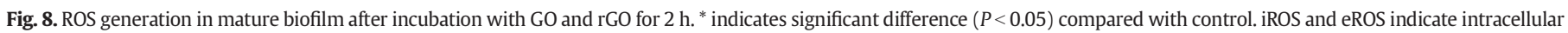
ROS and extracellular ROS. 


\section{Appendix A. Supplementary data}

Supplementary data to this article can be found online at http://dx. doi.org/10.1016/j.scitotenv.2016.12.093.

\section{References}

Ahmed, F., Rodrigues, D.F., 2013. Investigation of acute effects of graphene oxide on wastewater microbial community: a case study. J. Hazard. Mater. 256, 33-39.

Akhavan, O., Ghaderi, E., 2010. Toxicity of graphene and graphene oxide nanowalls against bacteria. ACS Nano 4, 5731-5736.

Akhavan, O., Ghaderi, E., 2012. Escherichia coli bacteria reduce graphene oxide to bactericidal graphene in a self-limiting manner. Carbon 50, 1853-1860.

Akhavan, O., Ghaderi, E., Esfandiar, A., 2011. Wrapping bacteria by graphene nanosheets for isolation from environment, reactivation by sonication, and inactivation by near-infrared irradiation. J. Phys. Chem. B 115, 6279-6288.

Avouris, P., 2010. Graphene: electronic and photonic properties and devices. Nano Lett. 10, 4285-4294.

Carpio, I.E.M., Santos, C.M., Wei, X., Rodrigues, D.F., 2012. Toxicity of a polymer-graphene oxide composite against bacterial planktonic cells, biofilms, and mammalian cells. Nanoscale 4, 4746-4756.

Chen, H.Q., Gao, D., Wang, B., Zhao, R.F., Guan, M., Zheng, L.N., Zhou, X.Y., Chai, Z.F., Feng, W.Y., 2014. Graphene oxide as an anaerobic membrane scaffold for the enhancement of $B$. adolescentis proliferation and antagonistic effects against pathogens $E$. coli and $S$. aureus. Nanotechnology 25, 165101.

Combarros, R., Collado, S., Díaz, M., 2016. Toxicity of graphene oxide on growth and metabolism of Pseudomonas putida. J. Hazard. Mater. 310, 246-252.

Davies, D., 2003. Understanding biofilm resistance to antibacterial agents. Nat. Rev. Drug Discov. 2, 114-122.

Diao, Y., Xin, Y.Q., Zhou, Y., Li, N., Pan, X.L., Qi, S.M., Qi, Z.L., Xu, Y.M., Luo, L., Wan, H.G., Lan, L., Yin, Z.M., 2014. Extracellular polysaccharide from Bacillus sp. strain LBP32 prevents LPS-induced inflammation in RAW 264.7 macrophages by inhibiting NF- $\kappa B$ and MAPKs activation and ROS production. Int. Immunopharmacol. 18, 12-19.

Djordjevic, D., Wiedmann, M., McLandsborough, L., 2002. Microtiter plate assay for assessment of Listeria monocytogenes biofilm formation. Appl. Environ. Microbiol. 68 2950-2958.

Ferry, J.L., Craig, P., Hexel, C., Sisco, P., Frey, R., Pennington, P.L., Fulton, M., Geoff, S.I., Decho, A.W., Kashiwada, S., Murphy, C.J., Shaw, T.J., 2009. Transfer of gold nanoparticles from the water column to the estuarine food web. Nat. Nanotechnol. 4, 441-444.

Flemming, H.C., Neu, T.R., Wozniak, D.J., 2007. The EPS matrix: the "house of biofilm cells". J. Bacteriol. 189, 7945-7947.

Goenka, S., Sant, V., Sant, S., 2014. Graphene-based nanomaterials for drug delivery and tissue engineering. J. Control. Release 173, 75-88.

Gurunathan, S., Han, J.W., Dayem, A.A., Eppakayala, V., Kim, J.H., 2012. Oxidative stressmediated antibacterial activity of graphene oxide and reduced graphene oxide in Pseudomonas aeruginosa. Int. J. Nanomedicine 7, e14.

Heydorn, A., Nielsen, A.T., Hentzer, M., Sternberg, C., Givskov, M., Ersbøll, B.K., Molin, S., 2000. Quantification of biofilm structures by the novel computer program COMSTAT. Microbiology 146, 2395-2407.

Hu, X., Zhou, Q., 2013. Health and ecosystem risks of graphene. Chem. Rev. 113, 3815-3835.

Hu, W., Peng, C., Luo, W., Lv, M., Li, X., Li, D., Huang, Q., Fan, C.H., 2010. Graphene-based antibacterial paper. ACS Nano 4, 4317-4323.

Hui, L., Piao, J.G., Auletta, J., Hu, K., Zhu, Y., Meyer, T., Liu, H.T., Yang, L.H., 2014. Availability of the basal planes of graphene oxide determines whether it is antibacterial. ACS Applied Materials \& Interfaces 6, 13183-13190.

Kamat, J.P., Devasagayam, T.P.A., Priyadarsini, K.I., Mohan, H., 2000. Reactive oxygen species mediated membrane damage induced by fullerene derivatives and its possible biological implications. Toxicology 155, 55-61.

Kemp, K.C., Seema, H., Saleh, M., Le, N.H., Mahesh, K., Chandra, V., Kim, K.S., 2013. Environmental applications using graphene composites: water remediation and gas adsorption. Nanoscale 5, 3149-3171.
Krishnamoorthy, K., Veerapandian, M., Zhang, L.H., Yun, K., Kim, S.J., 2012. Antibacterial efficiency of graphene nanosheets against pathogenic bacteria via lipid peroxidation. J. Phys. Chem. C 116, 17280-17287.

Kumar, S., Raj, S., Kolanthai, E., Sood, A.K., Sampath, S., Chatterjee, K., 2015. Chemica functionalization of graphene to augment stem cell osteogenesis and inhibit biofilm formation on polymer composites for orthopedic applications. ACS Appl. Mater. Interfaces 7, 3237-3252.

Liu, S., Zeng, T.H., Hofmann, M., Burcombe, E., Wei, J., Jiang, R., Kong, J., Chen, Y., 2011. Antibacterial activity of graphite, graphite oxide, graphene oxide, and reduced graphene oxide: membrane and oxidative stress. ACS Nano 5, 6971-6980.

Liu, L., Zhu, C., Fan, M., Chen, C., Huang, Y., Hao, Q., Yang, J.Z., Wang, H.Y., Sung, D.P., 2015. Oxidation and degradation of graphitic materials by naphthalene-degrading bacteria. Nanoscale 7, 13619-13628.

Lyon, D.Y., Alvarez, P.J., 2008. Fullerene water suspension ( $\left.\mathrm{nC}_{60}\right)$ exerts antibacterial ef fects via ROS-independent protein oxidation. Environ. Sci. Technol. 42, 8127-8132.

Ocsoy, I., Paret, M.L., Ocsoy, M.A., Kunwar, S., Chen, T., You, M., Tan, W.H., 2013. Nanotechnology in plant disease management: DNA-directed silver nanoparticles on graphene oxide as an antibacterial against Xanthomonas perforans. ACS Nano 7, 8972-8980.

Olson, M.E., Ceri, H., Morck, D.W., Buret, A.G., Read, R.R., 2002. Biofilm bacteria: formation and comparative susceptibility to antibiotics. Can. J. Vet. Res. 66, 86-92.

Órpez, R., Martínez, M.E., Hodaifa, G., El Yousfi, F., Jbari, N., Sánchez, S., 2009. Growth of the microalga Botryococcus braunii in secondarily treated sewage. Desalination 246, 625-630.

O'Toole, G., Kaplan, H.B., Kolter, R., 2000. Biofilm formation as microbial development. Annu. Rev. Microbiol. 54, 49-79.

Perreault, F., de Faria, A.F., Nejati, S., Elimelech, M., 2015. Antimicrobial properties of graphene oxide nanosheets: why size matters. ACS Nano 9, 7226-7236.

Qiu, Y., Wang, Z., Owens, A.C., Kulaots, I., Chen, Y., Kane, A.B., Hurt, R.H., 2014. Antioxidant chemistry of graphene-based materials and its role in oxidation protection technology. Nanoscale 6, 11744-11755.

Rodrigues, D.F., Elimelech, M., 2010. Toxic effects of single-walled carbon nanotubes in the development of E. coli biofilm. Environmental Science \& Technology 44 4583-4589.

Ruiz, O.N., Fernando, K.S., Wang, B., Brown, N.A., Luo, P.G., McNamara, N.D., Vangsness, M., Sun, Y.P., Bunker, C.E., 2011. Graphene oxide: a nonspecific enhancer of cellular growth. ACS Nano 5, 8100-8107.

Salas, E.C., Sun, Z., Luttge, A., Tour, J.M., 2010. Reduction of graphene oxide via bacterial respiration. ACS Nano 4, 4852-4856.

Starkey, M., Parsek, M.R., Gray, K.A., Chang, S.I., 2004. A sticky business: the extracellular polymeric substance matrix of bacterial biofilms. In: Ghannoum, M., O'Toole, G. (Eds.), Microbial Biofilms. ASM Press, Washington, DC, pp. 174-191.

Stewart, P.S., Costerton, J.W., 2001. Antibiotic resistance of bacteria in biofilms. Lancet $358,135-138$.

Takahashi, N., Ishihara, K., Kato, T., Okuda, K., 2007. Susceptibility of Actinobacillus actinomycetemcomitans to six antibiotics decreases as biofilm matures. J. Antimicrob. Chemother. 59, 59-65.

Tu, Y., Lv, M., Xiu, P., Huynh, T., Zhang, M., Castelli, M., Liu, Z.R., Huang, Q., Fan, C.H., Fang H.P., Zhou, R.H., 2013. Destructive extraction of phospholipids from Escherichia col membranes by graphene nanosheets. Nat. Nanotechnol. 8, 594-601.

Vecitis, C.D., Zodrow, K.R., Kang, S., Elimelech, M., 2010. Electronic-structure-dependent bacterial cytotoxicity of single-walled carbon nanotubes. ACS Nano 4, 5471-5479.

Wei, X.Q., Hao, L.Y., Shao, X.R., Zhang, Q., Jia, X.Q., Zhang, Z.R., Lin, Y.F., Peng, Q., 2015. Insight into the interaction of graphene oxide with serum proteins and the impact of the degree of reduction and concentration. ACS Applied Materials \& Interfaces 7. $13367-13374$

Zhang, P., Zhang, R., Fang, X., Song, T., Cai, X., Liu, H., Du, S.T., 2016. Toxic effects of graphene on the growth and nutritional levels of wheat (Triticum aestivum L.): short-and long-term exposure studies. J. Hazard. Mater. 317, 543-551.

Zou, X., Zhang, L., Wang, Z., Luo, Y., 2016. Mechanisms of the antimicrobial activities of graphene materials. J. Am. Chem. Soc. 138, 2064-2077. 